\title{
SELF-SIMILAR TRAFFIC GENERATION USING A FRACTIONAL ARIMA MODEL - APPLICATION TO VBR MPEG VIDEO TRAFFIC
}

\author{
Luis J. de la Cruz, Esteve Pallares, Juan J. Alins and Jorge Mata \\ Department of Applied Mathematics and Telematics \\ Polytechnic University of Catalonia \\ C/ Jordi Girona 1 i 3, Modul C3, Campus Nord \\ 08034 - Barcelona - SPAIN \\ phone : +34-93-4016014 fax : +34-93-4015981 \\ ljcruz@mat.upc.es, jmata@mat.upc.es
}

\begin{abstract}
Resumo - Medidas de tráfego e estudos recentes têm demonstrado que o tráfego de vídeo com codificação de taxa variável (VBR), assim como o tráfego de redes locais (LAN), apresentam dependências temporais de longa duração. Quando este tipo de tráfego é transmitido por redes ATM, esta dependência longa implica na necessidade de maiores "buffers" e capacidades de canal. Neste trabalho, utiliza-se um modelo ARIMA fracionário para representar tráfego de vídeo VBR com codificação MPEG. A componente fracionária integrada do modelo é baseada em um processo chamado de "diferenças fracionárias". O modelo foi obtido de três sequiências longas de tráfego, e tem sido usado para sintetizar seqüências artificiais de tráfego com a desejada dependência temporal de longa duração.

Abstract - Recent studies and traffic measurements have shown that variable bit rate (VBR) video traffic, as well as local area networks (LAN) traffic, exhibits long range dependence (LRD). When this kind of traffic is transmitted over ATM networks, the LRD property implies a bigger necessity in term of buffer allocation and channel capacity. In this paper, a new fractional ARIMA model for VBR MPEG video traffic at frame level is presented. The fractional integrative component of the model is based on a process called "fractional differencing". The model has been obtained from three very long sequences. It has been used to synthesize artificial traffic, which presents the desired LRD.
\end{abstract}

Keywords - Self-similar traffic, VBR MPEG video traffic and Fractional ARIMA models.

\section{INTRODUCTION}

Local area networks (LAN) and variable bit rate (VBR) video traffics exhibits long range dependence (LRD) in their autocorrelation function [1][2][3]. This kind of traffic has been called self-similar traffic, because it presents a "fractal" behavior. The LRD property give rise to two main problems:

- A bigger need of buffers and channels capacity to maintain a specified quality of service (QoS), and

- a lost in the statistical multiplexing gain when this traffic is transmitted over ATM networks.
The first problem is analyzed in [4] by means of simulations. The results show the increase of the queue lengths in the multiplexers when the input traffic presents LRD. On the other hand, a very good study about the second problem is carried out in [5]. Another recent work [6] deals with special situations in which LRD does not imply these problems.

This paper proposes a new model to capture the VBR MPEG video traffic [7]. The model is derived from an autoregressive, integrative and moving average (ARIMA) process [8]. The integrative component is fractional, giving rise to the LRD of the synthesized traffic. This process has been called "fractional differencing". In a previous work, an ARIMA model without LRD was presented [9]. Its capacity of prediction, as well as its application in traffic shaping, was contrasted in [10].

The objectives of the present study can be summarized as follows:

- To study and to validate a way of implementing the fractional differencing process,

- to obtain a new fractional ARIMA model for VBR MPEG video traffic at frame level,

- to study some traces of generated traffic, in order to validate the model.

The rest of the paper is organized as follows. In the next three sections, a brief review of MPEG video traffic, ARIMA processes and self-similar processes is presented. The fractional differencing method is also introduced. Section 5 is focussed on the fractional ARIMA model fit. In Section 6, a study and discussion about the generated traffic is presented. Finally, Section 7 presents the conclusions of the paper.

\section{MPEG VIDEO TRAFFIC}

The MPEG coding algorithm was developed to achieve a high compression ratio with a good picture quality. MPEG can be used to transmit real-time variable bit rate broadcast video and it is suitable for video-on-demand in ATM networks.

MPEG has two main coding modes: interframe mode and intraframe mode (I). In its turn, two types of frames 
can be distinguished by the interframe mode, predicted (P) and bidirectionally-predicted (B) frames. The Intra coded frames (I) are coded without any reference to other frames. Predictive coded frames $(\mathrm{P})$ are coded using motion compensated prediction from a past I or $\mathrm{P}$ frame. This implies a more efficient coding. Bidirectionally-predicted coded frames (B) provide the highest degree of compression using the previous and next I or $\mathrm{P}$ as a reference. A video sequence of pictures (SOP) is divided into groups of $\mathrm{N}$ pictures (GOP). A GOP consists of subgroups of M pictures where the first is a reference picture, intra or predicted, and the rest are bidirectionally-predicted. The image quality depends on the values $\mathrm{M}, \mathrm{N}$ and the selected quantizer step size $(\mathrm{Q})$.

Four levels of coding can also be considered: picture, slice, macroblock and block. A picture (or frame) is a basic unit of display. The frame size in pixels depends on the application. A slice is a horizontal strip within a frame. According to the MPEG-I standard, each frame is divided into slices of 16 pixels of width, which implies that the frames are divided into 18 slices. A macroblock consists of four $8 \times 8$ blocks of luminance pixels and two $8 \times 8$ chrominance blocks. The smallest unit is a block which is a $8 \times 8$ matrix of pixels.

MPEG codec can be set in an open-loop mode to maintain the subjective quality with a fixed $Q$, and the coded variable bit rate (VBR) output stream is delivered to the network. A suitable choice of $\mathrm{Q}, \mathrm{M}, \mathrm{N}$ parameters is important to minimize the traffic bit rate for a fixed subjective quality or for a constant signal-to-noise ratio (SNR).

The variations of the bit rate generated in the codification are produced by intrinsic and extrinsic reasons. The changes of the complexity and activity of the sequence to be coded produce the extrinsic ones. The intrinsic reasons are related, fundamentally, to the codification modes applied on the frames. Thus, the I frames need a higher number of bits than the frames P or B because the I frames only exploit the spatial redundancy using the DCT transform technique. In addition, the $\mathrm{P}$ frames tend to generate greater number of bits than B ones, since only motion compensation is applied respect to the previous reference image. Within the codification of the frames, another factor that gives rise to variations of the generated bit rate is the exploitation of the entropy using runlength codes.

An example of a common pattern of the MPEG frame rates is shown in figure 1 ..

The extrinsic reasons that produce fluctuations in the bit rate depend on the content of the frames to code. The frames with greater grade of detail or greater texture have a high complexity level and reduce the efficiency of the spatial redundancy exploitation. The high activity scenes with fast camera movements, zooms and plane changes, avoid the use of the predictive compression technique. In this way, these scenes increase the bit rate with respect to smaller activity sequences.

In general, the coders do not deliver directly the traffic to the user interface because, usually, a smoothing system is enabled. The smoothing is carried out through a small storage buffer. The insertion of the buffer introduces a delay in the cells delivered to the network. The use of the smoothing allows to maintain a bit rate approximately constant during

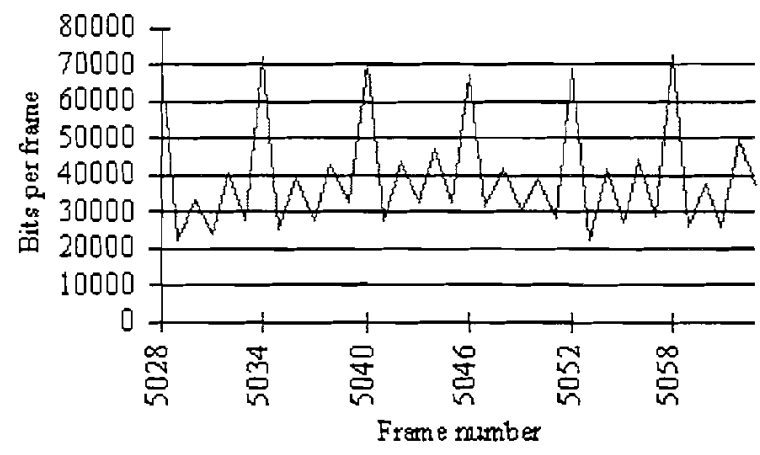

Figure 1. Classic pattern of the bits per frame generated by a VBR MPEG coder with $(\mathrm{Q}=6, \mathrm{M}=2, \mathrm{~N}=6)$ set parameters.

a time interval. The smoothing is applied to decrease the variability of the traffic and its peak rate. Likewise, the intrinsic periodic fluctuation of rate generated by the MPEG algorithm can be removed. In this way, the VBR MPEG traffic shaping allows to reduce the allocated resources on the virtual circuit. Moreover, the effects of the periodic peak rate arrivals to the multiplexers and switch fabrics are avoided. Therefore, the employment of the traffic shaping maximizes the statistical multiplexing gain.

Most of the studies are focused on the modeling and the prediction of the rate generated in a frame interval. The main reason is that the human perceptive system does not appreciate a delay less than $100 \mathrm{~ms}$ though it is admissible until $200 \mathrm{~ms}$ [1]. Therefore, the traffic shaper can introduce a delay of only several frames. At the same time, this delay allows to use the Bidirectionally-Predicted mode in the MPEG algorithm for interactive services.

Depending on the temporal requirements of the service all the generated cells for the GoP can be stored. Afterwards, the cells are delivered to the network at a constant rate during an interval of the same duration. For services with more restrictive temporal requirements is essential the reduction of the storage time (about $80 \mathrm{~ms}$ ). This reduction makes necessary the application of prediction techniques. These techniques permit the reduction of the traffic source burstiness and to satisfy the temporal constrains. The smoothing in intervals of duration one GoP allows to extract the intrinsic variations of the rate introduced by the MPEG algorithm. In this way, the rate generated only depends on the complexity and activity of the scenes.

\section{THE ARIMA PROCESSES}

These processes have been widely studied in the literature and in their more general form are denominated autoregressive, integrative, moving average processes (ARIMA) [8]. The autoregressive models are used in the context of sources of synthetic traffic. Also, these models can be employed to forecast the bit rates in intervals of fixed duration [14][15]. The ARIMA(p,d,q) models are decomposed in an autoregressive component of order $p$, an integrative component of order $d$ and a moving average 
component of order $\mathrm{q}$.

The autoregressive component reflects the dependence between the current generation and the last $\mathrm{p}$ generations. Thus, for an $\operatorname{AR}(\mathrm{p})$ process the values generated in a time series $\mathrm{Y}=(\mathrm{y} 0, \mathrm{y} 1, . ., \mathrm{yn})$ are obtained from the $\mathrm{p}$ past values and an independent factor from the times series. This factor can be modeled as a process with identically independent distributed values $\mathrm{W}=(\mathrm{w} 0, \mathrm{w} 1, . . \mathrm{wn})$. The time series $\mathrm{W}$ are denominated residual series. These time series are considered as the prediction error of the following generation of the process. Customarily, the values from the series $\mathrm{W}$ are synthesized as the realization of a gaussian random variable with an average and a standard deviation directly related with the corresponding moments of the process AR to generate. So that:

$$
y(n)=a_{1} y(n-1)+a_{2} y(n-2)+\ldots+a_{p} y(n-p)+w(n)
$$

where the terms $a_{i}$ are constant coefficients.

The $\mathrm{MA}(q)$ component of the process reflects the dependency in the generation of the past values of the independent process that contributes in the obtained value. In this way, a MA(q) process would be expressed as :

$$
\begin{aligned}
x(n) & =b_{0} w(n)+b_{1} w(n-1)+b_{2} w(n-2)+\ldots+ \\
& +b_{q}(n) w(n-q)
\end{aligned}
$$

where the terms $b_{i}$ are constant coefficients.

The integrative contribution allows to capture the non stationarity of the moments of the stochastic process. Although the integrative component can be considered within the AR component by its formulation, its synthesis depends on different factors. Thus, the integrative component also shows the dependence with past values of the series but its synthesis depends on the non stationary moments of the process. The order $d$ of the integrative component is fixed by the order of the highest non stationary moment of the stochastic process. In general, the integrative component can be expressed:

$$
s(n)=c_{1} s(n-1)+c_{2} s(n-2)+\ldots+c_{d} s(n-d)+w(n)
$$

where:

$$
c_{i}=\left(\begin{array}{c}
d \\
i
\end{array}\right)(-1)^{i+1} \quad i \in\{1,2, \ldots, d\}
$$

For example, a process whose mean is non-stationary and the rest of high order moments are stationaries would have an integrative component of order 1 . This integrative process is the so-called "random walk". If the behavior of the variance shows a clear trend during long intervals it is convenient to apply a transformation like the Box-Cox [8].

A scheme of the ARIMA model is shown in figure 2.. The interpretation of an ARIMA (p,d,q) process can be carried out defining the delay operator $z-1$ [1.3]. So that, the general expression of an ARIMA(p,d,q) process can be expressed by its $Z$ transform as:

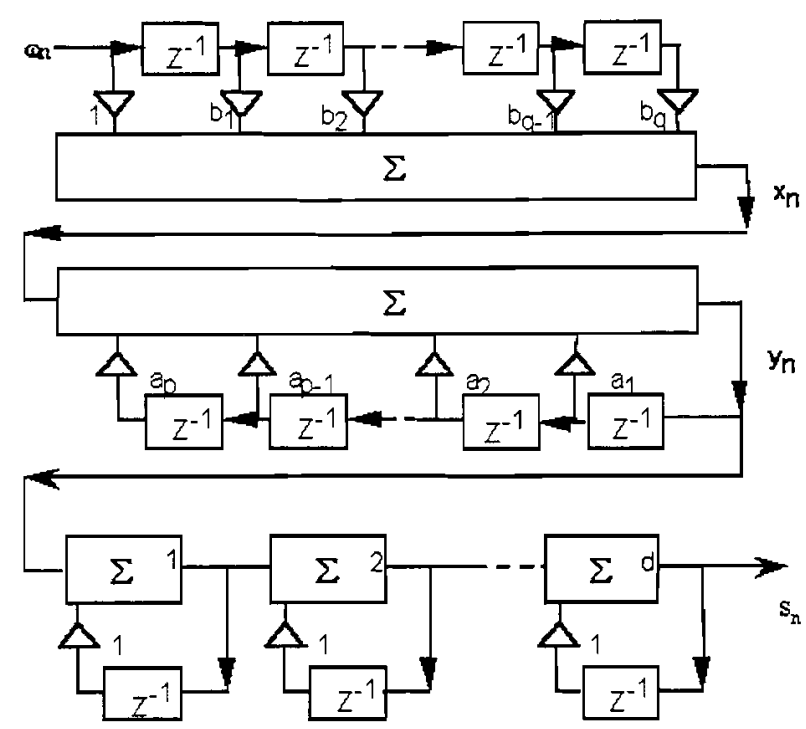

Figure 2. $\operatorname{ARIMA}(p, d, q)$ filter scheme.

$$
S(z)=\frac{B(z)}{A(z) C(z)} W(z)
$$

where:

$$
\begin{gathered}
Y(z)=Z(y)=\sum_{n=0}^{\infty} y_{n} z^{-n} \\
X(z)=Z(x)=\sum_{n=0}^{\infty} x_{n} z^{-n} \\
W(z)=Z(w)=\sum_{n=0}^{\infty} w_{n} z^{-n} \\
S(z)=Z(s)=\sum_{n=0}^{\infty} s_{n} z^{-n}
\end{gathered}
$$

and:

$$
\begin{gathered}
B(z)=\frac{X(z)}{W(z)} \quad A^{-1}(z)=\frac{Y(z)}{X(z)} \\
C^{-1}(z)=\frac{S(z)}{Y(z)}
\end{gathered}
$$

Understanding this expression as the relationship between the input $w(n)$ and the output $y(n)$ of a digital filter in a given instant $n$, the transfer function of the filter $H(z)$ could be define as:

$$
H(z)=\frac{S(z)}{W(z)}=\frac{B(z)}{A(z) C(z)}
$$

Note that the roots of the polynomial $B(z)$ correspond to the zeroes of the filter and the zeroes of $A(z)$ and $C(z)$ to the poles. According to the definition of the $c_{i}$ values expressed in (4), the integrative order defines the multiplicity of the pole in $z=1$. This pole generates the instability of impulsional response. The rest of obtained poles $z_{k}$ will be found in the unit circle $\left|z_{k}\right|<1$ of the $\mathrm{Z}$ plane. 


\section{SELF-SIMILAR PROCESSES}

In a recent work [5], the definition of the (second order) selfsimilar processes is summarized and proved in the following way: A process $\mathrm{X}$ is called exactly second-order self-similar with parameter $H=1-\beta / 2,0<\beta<1$, if its autocorrelation function is:

$$
\begin{aligned}
& r(k)=\frac{1}{2}\left[(k+1)^{2-\beta}-2 k^{2-\beta}+(k-1)^{2-\beta}\right] \\
& k=1,2, \ldots
\end{aligned}
$$

The parameter $\mathrm{H}$ is known as Hurst parameter, and it defines the level of LRD of the process. Its values are in the interval $[0.5,1)$. A value of $\mathrm{H}$ equal to 0.5 indicates the absence of LRD. Typical values of this parameter for VBR MPEG video traffic are around 0.8 [2]. Figure 3 shows the autocorrelation function $r(k)$ for three values of $\mathrm{H}$.

Realizations exhibiting LRD may be obtained through a process called fractional differencing [3], which is now briefly motivated. These process are derived from the pure integrative process, also called ARIMA $(0, \mathrm{~d}, 0)$ process, with $\mathrm{d}$ not necessarily integer. A pure integrative process has the following transfer function [13]:

$$
H(z)=\frac{1}{C(z)}=\left(1-z^{-1}\right)^{-d}
$$

This function can be developed in the following way:

$$
H(z)=\frac{1}{C(z)}=\sum_{i=0} t_{i} z^{-i}
$$

where the coefficients can be derived applying Taylor's series:

$$
t_{i}=(-1)^{i} \frac{(-d)(-d-1) \ldots(-d-i+1)}{i !}
$$

and they can be related recursively as follows:

$$
\begin{aligned}
& t_{0}=1 \\
& t_{i}=t_{i-1} \frac{(d+i-1)}{i}
\end{aligned}
$$

Moreover, this coefficients constitute the impulsional response $h(n)$ of the $\operatorname{ARIMA}(0, d, 0)$ filter. The relation between the Hurst parameter and $d$ is [3]:

$$
H=d+0.5
$$

Figure 4 represents these coefficients for different values of $H$. Figure 5 shows the equivalence between an $\operatorname{ARIMA}(0, d, 0)$ filter and an infinite order MA filter obtained using the Taylor's series discussed above.

Let be $i(n)$ an uncorrelated process at the input of the filter, and $o(n)$ the output of the same filter. Then:

$$
o(n)=i(n) * h(n)
$$

Thus, the autocorrelation functions of these process satisfies:

$$
r_{o o}(n)=r_{i i}(n) * r_{h h}(n)
$$

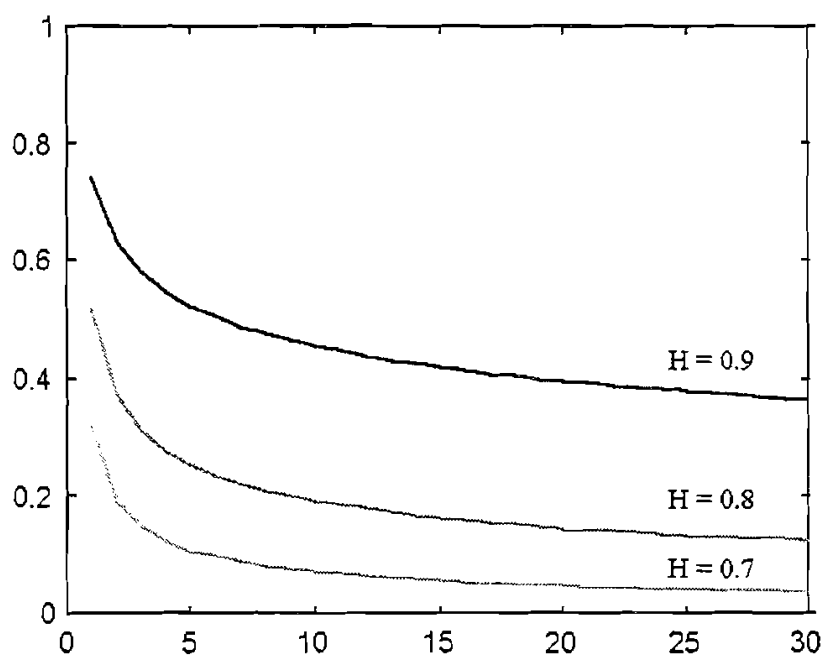

Figure 3. Autocorrelation function $r(k)$

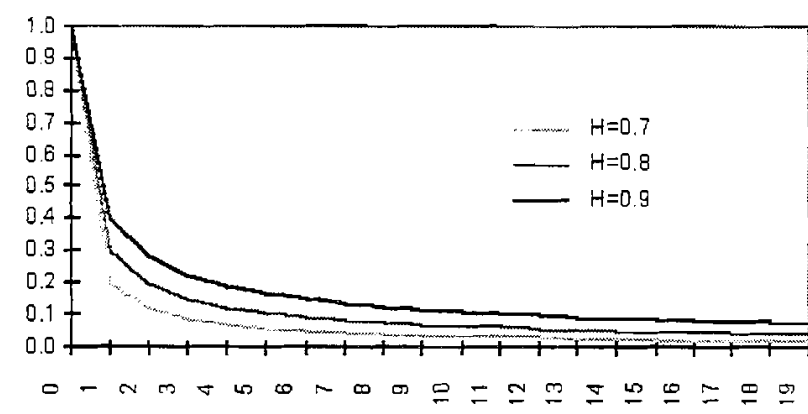

Figure 4. Filter coefficients.
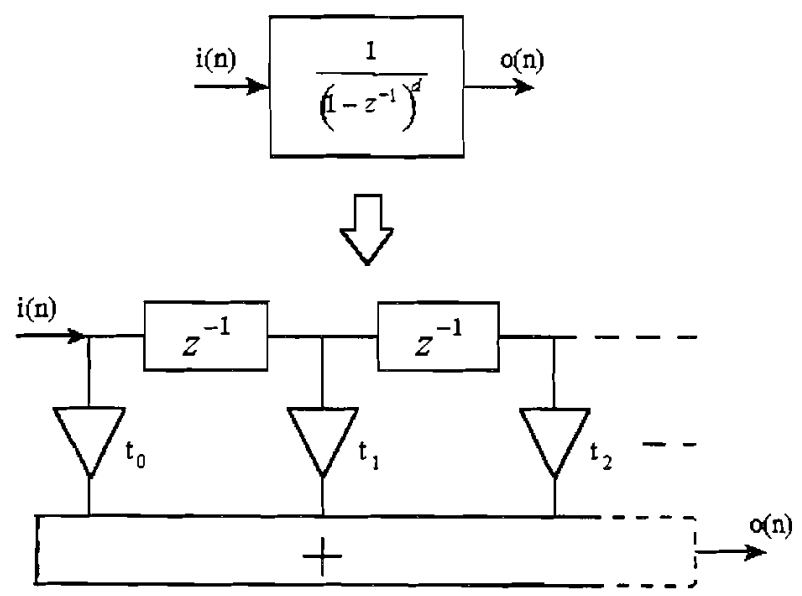

Figure 5. Equivalents filters. 


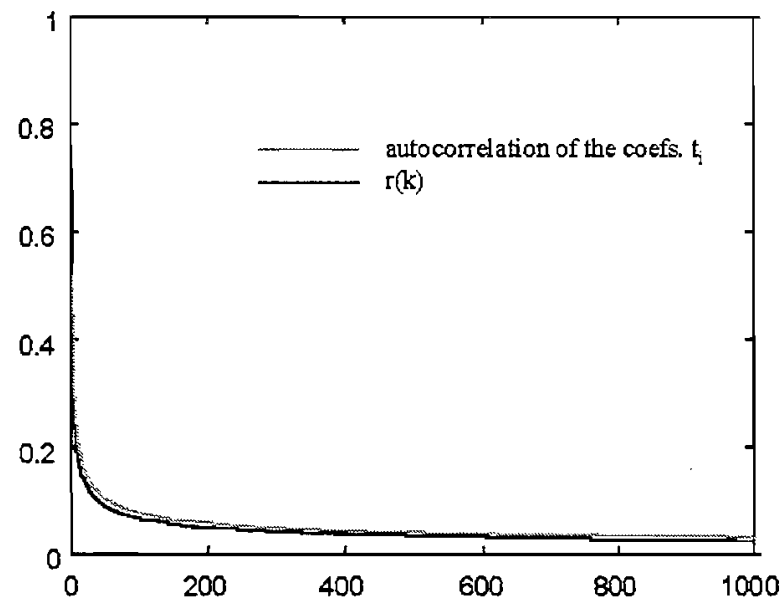

Figure 6. Autocorrelations comparison.

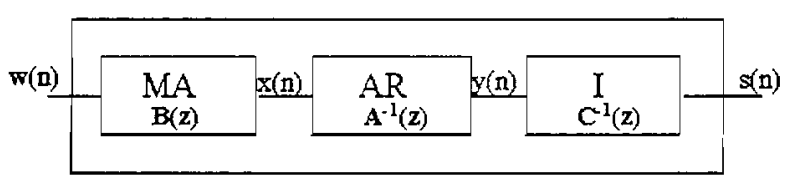

Figure 7. Components of an ARIMA process.

where $r_{x x}(n)$ represents the autocorrelation of the process $\mathrm{x}(\mathrm{n})$. The input is an uncorrelated signal, then:

$$
r_{i i}(n)=\delta(n)
$$

where $\delta(n)$ is the unit sample sequence [13]. Therefore:

$$
r_{o o}(n)=r_{h h}(n)
$$

In this way, the autocorrelation of the output is the same as the autocorrelation of the coefficients in expression (12). Moreover, the output of the filter is a second order selfsimilar process. Therefore, its autocorrelation must fit also the expression given in (9). In order to validate this study, in figure 6 the autocorrelation of 50000 coefficients is compared with the expression (9), for $H=0.8(d=0.3, \beta=0.4)$. One of the main goals of section 6 will be to obtain a valid bound for the minimum number of coefficients needed to obtain the desired LRD at the output of the filter.

\section{FRACTIONAL ARIMA MODEL}

In this section, a new fractional $\operatorname{ARIMA}(p, d, q)$ model is obtained for VBR MPEG video traffic at frame level. Figure 7 shows a general view of an ARIMA model.

To obtain the model, three long sequences has been used: "Jurassic Park" (175000 frames), "America" (34000 frames), and "Midnight murders" (40000 frames).

In order to determine which are the values of the AR components and MA, it is necessary to extract the integrative component of the actual process $s(n)$. According to the scheme presented in figure 7 , the residual ARMA series $y(n)$ 18

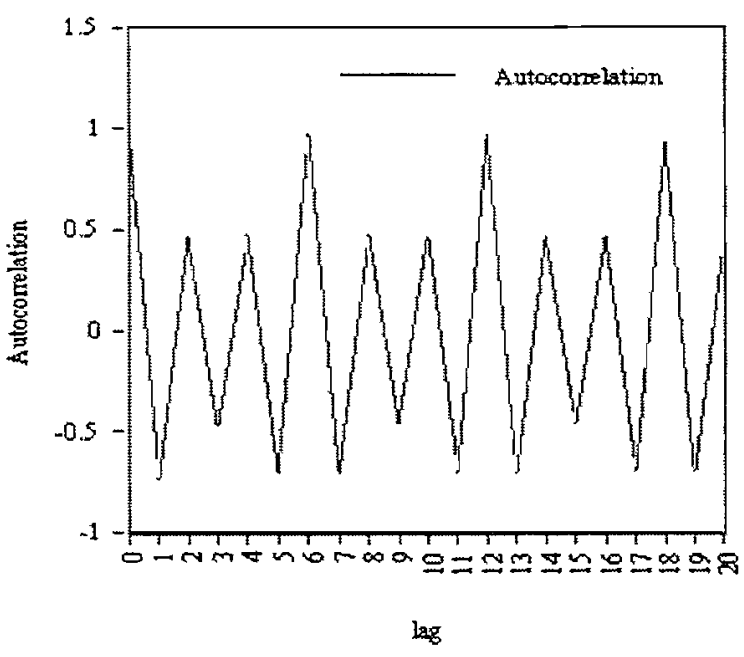

Figure 8. Autocorrelation function of the extracted integrative component time series.

and the real series $\mathbf{s}(\mathbf{n})$ are related as follows:

$$
Y(z)=C(z) S(z)=\left(1-z^{-1}\right)^{d} S(z)
$$

In this way, the temporal series $y(n)$ will be obtained at the output of a FIR filter, whose transfer function is (1 $\left.z^{-1}\right)^{d}$, when it is excited with the temporal series generated by the coder. It can be checked that the temporal series $\mathrm{y}(\mathrm{n})$ is a stochastic process with mean 0 and an invariant autocorrelation coefficients. This statistical analysis has been carried out with the three sequences using blocks of 15000 frames and with autocorrelation lags of 100 units. The probability distribution function fits a gaussian distribution in all cases. The difference is noted in the three temporal series on the standard deviation. This dissimilarity is related with the variability and complexity of the sequences.

The temporal series $y(n)$ presents a seasonal behavior [8] of period $\mathrm{N}=6$ according to the parameter chosen in the MPEG algorithm. This seasonal characteristic is reflected in the autocorrelation function shown in figure 8. Using the peaks of the autocorrelation function, which appear in multiples of $\mathrm{N}$, the $\mathrm{AR}$ component can be synthesized. To determine the coefficients of the AR component Least Squares estimation has been employed. The order of the seasonal model found is 2 . Hence, the transfer function of the AR component can be expressed with the calculated coefficients as:

$$
A(z)=1-0.626 z^{-6}-0.342 z^{-12}
$$

The MA component can be analyzed when the AR component of the $y(n)$ series is withdrawn. Applying the above explained technique for the integrative component the $x(n)$ series can be derived using the relation between the $y(n)$ and $x(n)$ series:

$$
X(z)=A(z) Y(z)
$$

Using a FIR filter with transfer function $\mathrm{A}(\mathrm{z})$ the series $\mathrm{x}(\mathrm{n})$ can be obtained at the output of this filter when the 


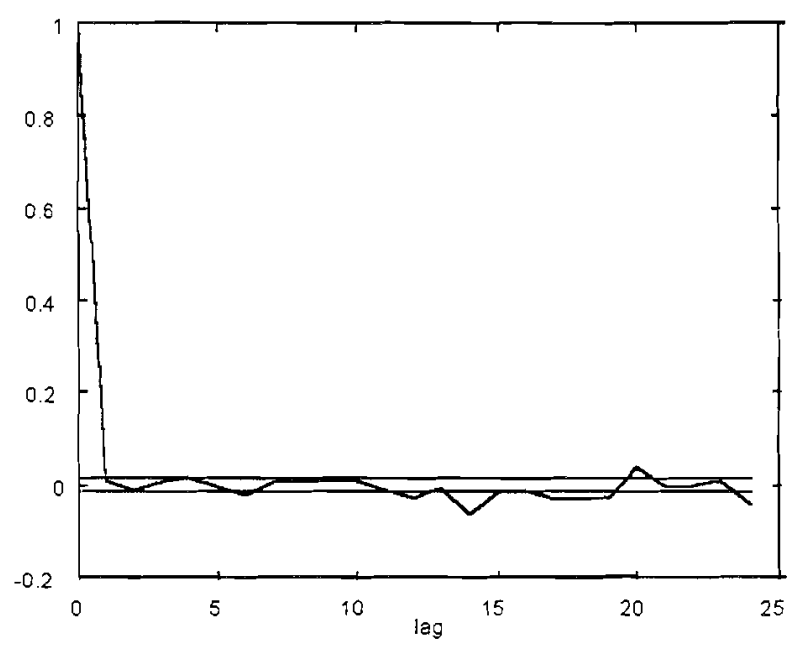

Figure 9. Correlation function of residuals.

$y(n)$ is applied at the input. To estimate the parameters of the MA process, least square estimation is applied to fit the partial autocovariance function of $\mathrm{x}(\mathrm{n})$. The best adjustment is obtained with a MA process with the following associated transfer function:

$$
\begin{aligned}
B(z)= & 1-0.0751 z^{-1}+0.221 z^{-2}+0.0039 z^{-3} \\
& +0.1816 z^{-4}-0.0149 z^{-5}-0.0599 z^{-6} \\
& -0.0254 z^{-7}+0.0500 z^{-8}-0.0286 z^{-9} \\
& +0.0189 z^{-10}-0.026 z^{-11}-0.1762 z^{-12} \\
& -0.0116 z^{-13}
\end{aligned}
$$

Finally, the ARIMA model can be expressed by the addition of the different components as:

$$
H(z)=\frac{S(z)}{W(z)}=\frac{B(z)}{A(z) C(z)}
$$

In order to evaluate the behavior of the above transfer function, an analysis of the forecast errors has been done for all sequences. Figure 9 presents the residuals autocorrelation and the $99 \%$ confidence intervals for the "America" sequence. The residual diagnostic determines that the forecast errors are very uncorrelated. Therefore, the ARIMA model allows a good prediction of the VBR MPEG traffic at frame level.

\section{TRAFFIC TRACES GENERATION}

The obtained model is used in this section to synthesize traffic. The main goal here is to obtain a minimum bound for the number of coefficients needed in the integrative component in order to achieve the desired LRD.

Thus, at the input of the model a gaussian noise, with zero mean and variance equal to 1 , was applied. The number of coefficients in the integrative component was increased from 100 to 50000 coefficients. The chosen value for $\mathrm{H}$ was 0.8 $(\mathrm{d}=0.3)$. The LRD level obtained in each case was measured using the test of variances, which is now briefly motivated [2]. For LRD processes, the variance of the aggregated series behaves for large $m$ like:

$$
\operatorname{Var}\left(X^{(m)}\right) \approx m^{-\beta} \sigma_{x}^{2}
$$

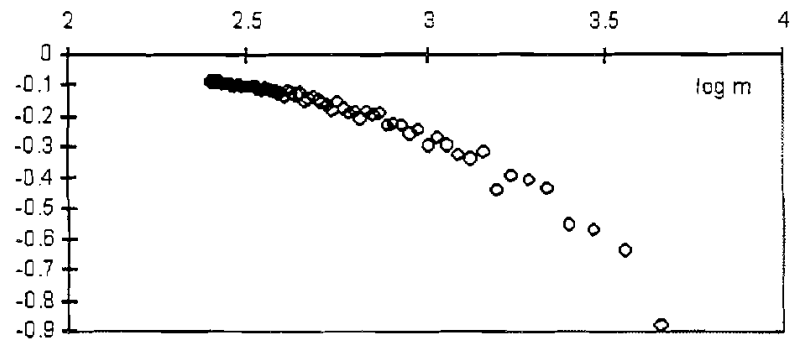

Figure 10. Variance-time plot for a generated trace.

where:

$$
\begin{aligned}
& 0<\beta<1 \\
& X^{(m)}=\left(X_{k}^{(m)}: k=1,2, \ldots\right) \\
& X_{k}^{(m)}=\frac{1}{m}\left(X_{k m-m+1}+\ldots+X_{k m}\right) \\
& k=1,2, \ldots
\end{aligned}
$$

The "variance-time" plot is a graphical method for distinguishing between short and long range dependence. To estimate $\beta$, which is related to $\mathrm{H}$ by $\beta=2-2 H$, the normalized variance of the aggregated series $\operatorname{Var}\left(X^{(m)}\right) / \sigma_{x}^{2}$ is plotted as a function of block size $\mathrm{m}$, on log-log coordinates, yielding $\beta$ as the limiting slope as $m \rightarrow \infty$. An example is shown in Figure 10.

In Table 1, the main results of these tests are presented. With 10000 coefficients, the obtained value for $\mathrm{H}, 0.782$, is very near of the expected value 0.8 . Even 5000 coefficients can be enough for some applications.

\begin{tabular}{|c|c|}
\hline Number of coefficients & Hurst parameter \\
\hline \hline 100 & 0.598 \\
\hline 500 & 0.657 \\
\hline 1000 & 0.714 \\
\hline 2000 & 0.747 \\
\hline 3000 & 0.761 \\
\hline 4000 & 0.767 \\
\hline 5000 & 0.771 \\
\hline 10000 & 0.782 \\
\hline 25000 & 0.783 \\
\hline
\end{tabular}

Table 1. Hurst parameter vs. Number of coefficients

In order to use these traces for simulations, the mean and the variance must be adjusted to typical values of VBR MPEG video traffic. For the "Jurassic Park" sequence, the mean is 43925 bits per frame, and the standard deviation is 31517 . Once adjusted with these values, a new set of traces was obtained. The autocorrelation function of one of these traces (with 10000 coefficients) and the autocorrelation function of the original "Jurassic Park" sequence are compared in figure 11 . The seasonal behavior presented in both functions was extracted for the sake of clarity. The slope of both functions for large lags, which is related with the LRD level, is very similar. This allows to test the goodness-of-fit.

The previous study allows to propose the model in Figure 12 for VBR video traffic generation. The output of the 


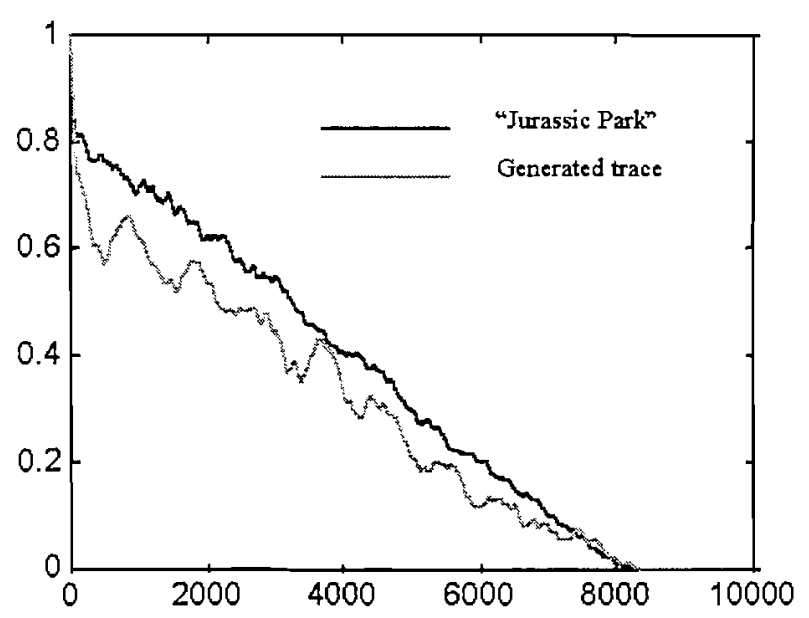

Figure 11. Generated trace and "Jurassic Park" autocorrelation functions.

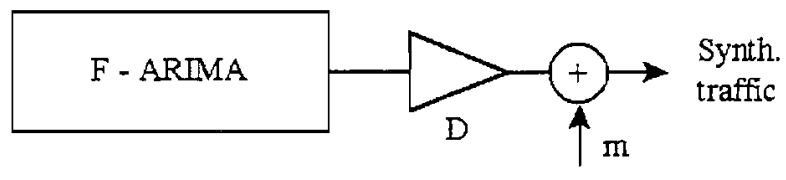

Figure 12. VBR video traffic generation.

fractional ARIMA model is amplified with a $D$ value to adjust the standard deviation. A big value of $\mathrm{D}$ give rises to a very variable traffic, modeling a video sequence with high level of complexity. On the contrary, a lower value models a video source with low complexity. Finally, the mean of the process is increased by a $\mathrm{m}$ value. This value can be used to set different image qualities of the MPEG codification.

In [11] and [12], two previous models to generate long range dependence have been presented. The first one deals with the Hosking's algorithm. The main disadvantage of this model is the need of storing all the previous samples in to generate a new one. Thus, this algorithm needs a large and variable amount of memory. Moreover, the time needed to generate every new sample is high and variable, with a strong computational load. However, the model presented in this work needs a little and constant amount of memory. The time wasted to generate every new sample is constant and negligible. Thus, it can be used to drive simulations on "real time". On the other hand, the "random midpoint displacement algorithm" is presented in [12]. Nevertheless, this algorithm can not be used to synthesize traffic "on line" for driving simulations, because it must generate the complete set of samples before the generated traffic can be used.

\section{CONCLUSIONS}

The self-similar nature of the VBR MPEG video traffic has been shown in several works. This property is shown in the long range dependence (LRD) of the autocorrelation function. Generally, it implies the need of a larger amount of 20 resources when this traffic is transmitted over ATM networks.

In this paper, a new model for this kind of traffic has been presented. It is a fractional ARIMA model, and it is based on the fractional differencing method. Using this construction, an equivalent filter with a finite number of coefficients has been used.

In order to validate the truncated order filter, it has been used to generate artificial traces of traffic. Each trace has been synthesized using a different number of coefficients. To obtain the LRD achieved in the synthesized traffic, the traces have been analyzed using the "variances test". With this test, the minimum number of coefficients needed to obtain a specified LRD has been found around 5000. This number can be even lower for some applications. Moreover, the autocorrelation function of the artificial traffic has been compared with the real traffic one, showing the obtained goodness-of-fit.

Finally, the fractional ARIMA model has been proposed to synthesize self-similar VBR MPEG video traffic.

\section{ACKNOWLEDGEMETNS}

This work has been developed using the Global ATM Standard Simulator and supported by the SIGLA Project (Spanish CICYT, TEL 96-1452).

\section{REFERENCES}

[1] M. W. Garret, "Contributions Toward Real-Time Services on Packet-Switched Networks", Ph.D. Dissertation CU/CTR/TR 340-93-20, Columbia University, New York, N.Y., May 1993.

[2] M.W. Garret and W. Willinger, "Analysis, Modeling and Generation of Self-Similar VBR Video Traffic", Proceedings of ACM SIGComm, pp. 269-280, 1994.

[3] W.E. Leland, M.S. Taqqu, W. Willinger and D.V. Wilson, "On the Self-Similar Nature of Ethernet Traffic", Proceedings of ACM SIGComm, pp. 183-193, 1993.

[4] A. Adas and A.Mukherjee, "On Resource Management and QoS Guarantees For Long Range Dependent Traffic", Proceedings of IEEE INFOCOM'95, pp. 779787, 1995.

[5] B. Tsybakov and N. Georganas, "On Self-Similar Traffic in ATM Queues: Definitions, Overflow Probability and Cell Delay Distribution", IEEE/ACM Transactions on Networking, vol. 5, no. 3, pp. 397-409, 1997.

[6] K.R. Krishnan and G. Meempat, "Long-range dependence in VBR video streams and ATM traffic engineering", Performance Evaluation, vol. 30, pp. 45-56, 1997.

[7] P. Pancha and M. El Zarki, "MPEG Coding For Variable Bit Rate Video Transmission", IEEE Communications Magazine, vol. 32, no.5, pp. 54-66, May 1994. 
[8] G. E .P. Box, G. M. Jenkins and G. C. Reinsel, "Time Series Analysis", Prentice Hall, New Jersey, 1994.

[9] L. J. de la Cruz, J. J. Alins and J. Mata, "Prediction Techniques for VBR MPEG Traffic Shaping', Proceedings of IEEE GLOBECOM'97, vol. 3, pp. 1434-1439, 1997.

[10] L. J. de la Cruz, J. J. Alins and J. Mata, "Predictive Shaping for VBR MPEG Video Traffic Transmission over ATM Networks", Performance on Information and Communication Systems, pp. 216-233, Chapman\&Hall, London, 1998.

[11] J.R.M.Hoskings, "Modeling persistence in hydrological time series using fractional differencing", Water Resources Res., vol. 20, no. 12, pp. 1898-1908, 1984.

[12] W.C. Lau, A. Erramilli, J. L. Wang and W. Willinger, "Self-Similar Traffic Generation: The Random Midpoint Displacement Algorithm and Its Properties", Proceedings of IEEE INFOCOM'95, pp.466-472, 1995.

[13] J. G. Proakis and D. G. Manolakis, "Introduction to Digital Signal Processing", Macmillan Publishing Company, New York, 1988.

[14] Grunenfelder, Cosmas, Manthrope and OdinmaOkafor, "Characterization of Video Codecs as Autoregressive Moving Average Processes and Related Queueing System Performance," IEEE JSAC vol.9, no. 3, pp. 284-292, April 1991.

15] F. Yegenoglu, B. Jabbari and Y. Zhang, "Motion Classified Autoregresive Modeling of Variable Bit Rate Video", IEEE Transactions on Circuits and Systems for Video Technology, vol. 3, no. 1, pp. 42-53, February 1993.

Luis J. de la Cruz was born in Granada, Spain. He received the M.S. degree in Telecommunications Engineering in 1994, and the Ph.D. degree in 1999, both from the Polytechnic University of Catalonia (UPC), Barcelona, Spain. In 1994, he joined the Broadband Networks Modeling and Analysis Group at the Department of Applied Mathematics and Telematics of the UPC. His research interests include multimedia services modeling and optimal resources allocation on broadband networks. Currently, he works as a Lecturer in the Polytechnic University of Catalonia.

Esteve Pallares received the M.S. degree in Telecommunications Engineering in 1994 from the Polytechnic University of Catalonia, Barcelona, Spain. Currently he is with Broadband Networks Modeling and Analysis Group at the Department of Applied Mathematics and Telematics of the UPC. His research interests include multimedia services modeling and optimal resources allocation on broadband networks. He is currently a Lecturer at the Polytechnic University of Catalonia.
Juan J. Alins received the M.S. degree in Telecommunications Engineering in 1994 from the Polytechnic University of Catalonia, Barcelona, Spain. Currently he is with the Cybernetic Institute at the UPC. His main research field is control theory. $\mathrm{He}$ is also a Lecturer at the Polytechnic University of Catalonia.

Jorge Mata was born in Barcelona in 1965. He received the M.S. degree in Telecommunications Engineering in 1991, and the Ph.D. degree in 1996, both from the Polytechnic University of Catalonia (UPC), Spain. He is currently a research staff menber with the Broadband Networks Modeling and Analysis Group at the Department of Applied Mathematics and Telematics of the UPC. His research interests include network services to the home, multimedia applications, high-speed networks, traffic modeling and statistical performance analysis. Currently, he is an Associate Professor at the Polytechnic University of Catalonia. 